\title{
Erratum: Dynamic Multiferroicity of a Ferroelectric Quantum Critical Point [Phys. Rev. Lett. 122, 057208 (2019)]
}

\author{
K. Dunnett $\odot$, J.-X. Zhu, N. A. Spaldin, V. Juričić, and A. V. Balatsky
}

(Received 1 April 2020; published 23 April 2020)

DOI: 10.1103/PhysRevLett.124.169903

We correct an error in the calculation of the susceptibility close to the ferroelectric (FE) quantum critical point (QCP), noting that the main conclusions of the Letter, that is the enhancement of the susceptibility in the vicinity of the FE QCP and the resulting dynamical multiferroicity, are unchanged. The error occurs in our reported perturbative correction $\chi^{(2)}$ (bubble diagram), given by Eq. (8) in the Supplemental Material of the original Letter. Here we show that the approach is accompanied by a significantly enhanced susceptibility rather than the divergence reported in the original Letter.

We recalculate here the bubble contribution $\chi^{(2)}$ at the Matsubara frequency $i \omega$, the momentum $\mathbf{q}$ and at zero temperature $(T=0)$, which explicitly reads as

$$
\chi_{i j}^{(2)}(i \omega, \mathbf{q})=C^{2} \epsilon_{i k l} \epsilon_{j k l} I(i \omega, \mathbf{q})
$$

where

$$
I(i \omega, \mathbf{q})=\int_{\Omega, \mathbf{k}} \frac{(2 \Omega-\omega)^{2}}{\left(\omega_{\mathbf{k}}^{2}+\Omega^{2}\right)\left[\omega_{\mathbf{k}-\mathbf{q}}^{2}+(\Omega-\omega)^{2}\right]} .
$$

Here, $\int_{\Omega, \mathbf{k}} \equiv \int d \Omega d^{3} \mathbf{k} /(2 \pi)^{4}$ is the integral over the Matsubara frequency and momentum, $\omega_{\mathbf{k}}^{2}=a+b k^{2}$ is the dispersion of the soft phonon modes defined in Eq. (2) of the original Letter, and $C$ is a constant defined in Eq. (6) of the original Letter. The exact form of the bubble contribution as given by Eq. (1) implies that Eq. (8) of the Supplemental Material of the original Letter is incorrect, and we hereby correct it.

To this end, we now directly compute this bubble contribution, first in terms of the Matsubara frequency, and subsequently taking analytical continuation to the real frequency. The integral over the Matsubara frequency in Eq. (2) reads as

$$
I(i \omega, \mathbf{q})=\int \frac{d^{3} \mathbf{k}}{(2 \pi)^{3}} \frac{1}{\omega_{\mathbf{k}}}\left[1-\frac{\left(\omega_{\mathbf{k}}-\omega_{\mathbf{k}-\mathbf{q}}\right)^{2}}{\omega^{2}+\left(\omega_{\mathbf{k}}+\omega_{\mathbf{k}-\mathbf{q}}\right)^{2}}\right]=I(i \omega, \mathbf{q}=0)+q^{2} I^{(2)}(i \omega)+\mathcal{O}\left(q^{4}\right) .
$$

First, we take the limit $\mathbf{q}=0$, for which, after performing the momentum integral, we find

$$
I(i \omega, \mathbf{q}=0)=\frac{\Lambda^{2}}{4 \pi \sqrt{b}}\left[1-\frac{a}{2 b^{3 / 2} \Lambda^{2}}\left(\ln 2 b \Lambda+\frac{1}{\sqrt{b}} \ln a b\right)\right] .
$$

We therefore obtain an expression for the bubble contribution that is frequency independent and scales as the square of the momentum cutoff $\Lambda$. This is in contrast to the form in Eq. (9) of the Supplemental Material in the original Letter, which featured divergences in this response at particular frequencies. The bubble piece therefore gives the frequency independent background to the main contribution stemming from the "bare" susceptibility $\chi^{(1)}$ [Eqs. (3) and (4) in the original Letter]. This correction does not change the main conclusions of the original Letter, derived from the contribution $\chi^{(1)}(i \omega)$, regarding the enhancement of the susceptibility due to the fluctuating electric dipoles in the vicinity of the FE QCP.

For completeness, we also present the result for the leading contribution to the bubble diagram at a finite momentum, which was not discussed in the original Letter.

To this end, we take the leading (quadratic) term in the expansion in Eq. (2) over momentum, which, after performing the analytical continuation $i \omega \rightarrow \omega+i \delta$, becomes 


$$
I^{(2)}(i \omega \rightarrow \omega+i \delta)=I^{(21)}(i \omega \rightarrow \omega+i \delta)+I^{(22)}(i \omega \rightarrow \omega+i \delta),
$$

where

$$
\begin{gathered}
I^{(21)}(\omega)=-\mathrm{P} \cdot \mathrm{V} \cdot \int \frac{d^{3} \mathbf{k}}{(2 \pi)^{3}}\left(\frac{b^{2} k^{2}}{8 \omega_{\mathbf{k}}^{4}}\right) \frac{\omega_{\mathbf{k}}}{\omega_{\mathbf{k}}^{2}-\left(\frac{\omega}{2}\right)^{2}}, \\
I^{(22)}(\omega)=-i \pi \int \frac{d^{3} \mathbf{k}}{(2 \pi)^{3}}\left(\frac{b^{2} k^{2}}{16 \omega_{\mathbf{k}}^{4}}\right) \sum_{\tau= \pm} \tau \delta\left(\omega_{\mathbf{k}}-\tau \frac{\omega}{2}\right) .
\end{gathered}
$$

Here, $I^{(21)}(\omega) \equiv I^{(21)}(i \omega \rightarrow \omega+i \delta)$, and analogous for $I^{(22)}$, while P. V. denotes the principal value of the integral. Straightforward calculation then yields

$$
I^{(21)}(\omega)=-\frac{1}{16 \pi^{2} \sqrt{b}}\left\{\ln \left(\frac{2 \Lambda}{\sqrt{a}}\right)-\frac{4 a}{\omega^{2}}+\frac{1}{2}\left(1-\frac{4 a}{\omega^{2}}\right)^{3 / 2} \ln \left[\frac{\omega-\sqrt{\omega^{2}-4 a}}{\omega+\sqrt{\omega^{2}-4 a}}\right]-\frac{i \pi}{2}\left[\frac{\left(\omega^{2}-4 a\right)^{3 / 2}}{\omega^{3}}\right]\right\}
$$

while

$$
I^{(22)}(\omega)=-\frac{i}{32 \pi \sqrt{b}} \frac{\left(\omega^{2}-4 a\right)^{3 / 2}}{\omega^{3}} \Theta\left(\left|\frac{\omega}{2}\right|-\sqrt{a}\right),
$$

with $\Theta(x)$ being the Heaviside step function. 